\title{
Epidemiologia em Saúde Coletiva: reflexões sobre uma experiência pedagógica integrando pós-graduação e serviço de saúde
}

\author{
Juliana Gonçalves*; Angélica Teresa Nascimento de Medeiros**; Bárbara Cássia de Santana Farias \\ Santos***; Taiana Brito Menêzes****; Zilane Silva Barbosa de Oliveira***; Luiz Roberto Augusto \\ Noro $* * * * *$
}

\author{
Doutoranda em Saúde Pública, Programa de pós-graduação \\ em Saúde Pública, IAM-Fiocruz PE \\ $* *$ \\ Doutora em Saúde Coletiva, Programa de Pós-graduação em \\ Saúde Coletiva, UFRN \\ *** Mestre em Saúde Coletiva, Programa de Pós-graduação em \\ Saúde Coletiva, UFRN \\ **** Doutoranda em Saúde Coletiva, Programa de Pós-graduação \\ em Saúde Coletiva, UFRN \\ ***** Pós-doutorado em Educação, Universidade de Barcelona; \\ Professor do Departamento de Odontologia e do Programa \\ de Pós-graduação em Saúde Coletiva, UFRN
}

Recebido em 30/01/2017. Aprovado em 11/05/2017.

\section{RESUMO}

A Epidemiologia subsidia o planejamento em saúde configurando-se como conhecimento essencial na produção do conhecimento e na tomada de decisão. O objetivo do estudo foi refletir acerca de uma experiência pedagógica proporcionada pelo componente curricular Epidemiologia em Saúde Coletiva do Programa de Pós-graduação em Saúde Coletiva da Universidade Federal do Rio Grande do Norte. Trata-se de um relato de experiência desenvolvido baseando-se nos princípios teóricos da sistematização de experiências propostos por Holliday. A referida experiência foi centrada em estratégia pedagógica que vislumbrou a situação do serviço como lócus de construção de proposta de intervenção, permitindo ao aluno o protagonismo como elemento fundante de seu aprendizado. A articulação com o serviço e seus profissionais foi fundamental para a sinalização das propostas de intervenção. O desafio da integração entre universidade e serviço de saúde é elemento fundamental no avanço de políticas públicas de saúde, em especial na perspectiva dos cursos de pós-graduação responsáveis pela produção de novo conhecimento e pelo entendimento do serviço como cenário privilegiado para aprendizado do Sistema Único de Saúde.

Descritores: Epidemiologia. Educação de Pós-graduação. Sistema Único de Saúde. Serviços de Integração Docente-Assistencial. 


\section{INTRODUÇÃO}

A Saúde Coletiva, enquanto corrente de pensamento, movimento social e prática teórica, implica em um desafio paradigmático no sentido de consolidar o campo como prática de saúde com relevância acadêmica $^{1}$. Este campo científico e âmbito de práticas em permanente (re)construção visa superar dialeticamente a Saúde Pública convencional e o modelo biomédico, tendo se desenvolvido historicamente no Brasil a partir do anos 1970, fundando-se na interface das Ciências Naturais e das Ciências Humanas e Sociais com uma natureza epistemológica e uma prática política diferenciadas, constituindo-se em três núcleos de saberes: Política, Planejamento e Gestão de Sistemas de Saúde, Epidemiologia e Ciências Humanas e Sociais ${ }^{2}$.

$\mathrm{Na}$ esfera acadêmica, a Pós-graduação Stricto Sensu em Saúde Coletiva, por meio de suas pesquisas, foi uma forte ferramenta na busca de delimitar a área, reafirmar a potência desse campo e ganhar espaço cada vez mais significativo no meio acadêmico, subsidiando, inclusive as discussões sobre a prática ${ }^{3,4}$.

A Universidade Federal do Rio Grande do Norte (UFRN) iniciou em 1978 o Mestrado em Odontologia Social, o qual hoje se denomina Programa de Pós-graduação em Saúde Coletiva, composto pelo Mestrado com duas áreas de concentração (Saúde Coletiva e Odontologia) e pelo Doutorado com uma área de concentração (Saúde Coletiva). O Programa destaca em seu Projeto Pedagógico, dentre outros aspectos, a oferta de atividades didáticas comprometidas com a autonomia do aluno na busca de referencial teórico, assim como o exercício da reflexão e da criatividade como mecanismos de aprendizado e desenvolvimento de pesquisas ${ }^{5}$.

Diante dessa premissa, o componente curricular Epidemiologia em Saúde Coletiva traz em seu método pedagógico o incentivo a uma postura discente para ser protagonista de seu aprendizado, além de promover os estudos teóricos de acordo com as demandas dos serviços de saúde, o que se mostra coerente com a perspectiva de "empoderamento" do discente no que se refere a uma aprendizagem ativa e baseada em problemas ${ }^{6}$.

A Epidemiologia é a ciência que subsidia o planejamento em saúde a partir de informações coletadas de inquéritos populacionais ou da produção de serviços, configurando-se como conhecimento essencial na produção do conhecimento e na tomada de decisão. A Epidemiologia também tem papel de destaque na reorganização do setor saúde, buscando a integração entre a academia e os serviços de saúde pública ${ }^{7}$.

Entendendo a aproximação da Pósgraduação Stricto Sensu com a realidade dos serviços como ainda distante, o papel social da universidade na melhoria das condições de saúde da população e a necessidade de formar professores na área da saúde coletiva com capacidade para extrapolar o conhecimento meramente teórico, o objetivo do presente estudo foi refletir acerca de uma experiência pedagógica na perspectiva de um aprendizado investigativo, contextualizado e colaborativo, no qual a articulação entre academia e serviços pode desempenhar papel preponderante na definição de políticas públicas de saúde.

\section{RELATO DE EXPERIÊNCIA}

Trata-se de uma reflexão fundamentada nos princípios teóricos da sistematização de experiências $^{8}$. Sistematizar está intimamente relacionado com o exercício de experiências práticas concretas, ou seja, relaciona-se com uma proposta de refletir a experiência a partir de um aprofundamento na dinâmica das vivências, fundamentada na concepção metodológica dialética a qual vê a realidade como histórico- 
social; sensível à ação e transformação humana, trazendo como faces de uma mesma moeda a unidade e a mutabilidade, fazendo-se, portanto, contraditória.

A experiência ocorreu no componente curricular Epidemiologia em Saúde Coletiva, do Programa de Pós-graduação em Saúde Coletiva da Universidade Federal do Rio Grande do Norte. Para desenvolvimento das atividades pedagógicas foram definidos 8 momentos, distribuídos entre encontros presenciais e não presenciais.

Para descrição da experiência foram utilizados diários de campo dos proponentes do referido relato, projeto pedagógico do Programa de Pós-graduação em Saúde Coletiva da UFRN, plano de ensino e orientações de aula, apresentados no decorrer do componente curricular.

Baseado no conceito adotado de sistematização e guardando coerência com o método proposto ${ }^{8}$, o relato é apresentado em cinco momentos: o ponto de partida, as perguntas iniciais, recuperação do processo vivido; a reflexão de fundo; e os pontos de chegada.

\section{$O$ ponto de partida}

No primeiro encontro do componente curricular foi realizada a apresentação do plano de ensino e um diagnóstico inicial sobre o conhecimento prévio da turma. O referido diagnóstico consistiu em cada aluno expressar em uma palavra ou sentença o conteúdo da Epidemiologia que lhe despertava interesse de aprofundamento. As temáticas foram agrupadas conforme os termos que se repetiam e que se complementavam, formando grupos de trabalho que receberam como missão a elaboração de uma apresentação sobre este tema, os quais foram: história natural da doença, determinantes sociais da saúde, transição epidemiológica, riscos em saúde, indicadores de saúde, sistemas de informação em saúde, vigilância em saúde e Epidemiologia e serviços de saúde. Foi estimulada a utilização de, no mínimo, cinco artigos científicos para embasar a apresentação, dos quais dois deveriam ser socializados para a leitura dos demais da turma.

Ensinar exige pesquisa, não havendo pesquisa sem ensino, requerendo ainda respeito aos saberes dos educandos ${ }^{9}$. No contexto apresentado, a Epidemiologia perpassa a formação acadêmica dos diversos profissionais de saúde, implicando em uma formação básica da disciplina durante a graduação. Nesse sentido, esta proposta foi bem aceita pela turma, tanto pela ênfase ao estudo dos pontos de interesse como pelo incentivo à busca do conhecimento em fontes com maiores possibilidades de atualização e aprofundamento de temas dos quais já se tinha um conhecimento anterior.

No segundo momento do componente curricular foram realizadas as apresentações e discussões destes temas, de forma sequenciada e lógica, organizada pelo facilitador, conforme mencionadas, emergindo neste contexto os conceitos de Epidemiologia nos serviços de saúde. Seguindo esta dinâmica, foi proposta a formação de novos grupos de trabalho agrupados conforme as áreas de atuação dentro do programa. Os três grupos formados receberam o desafio de eleger uma Unidade de Saúde da Família (USF) do município de Natal para realização de visita e observação "in loco" sobre a utilização da Epidemiologia nos serviços de saúde.

Foi disponibilizado um roteiro que norteava as observações e articulações a serem realizadas na unidade. Contudo, os discentes possuíam a liberdade de fazer adaptações no roteiro, tendo em vista as peculiaridades existentes em cada unidade visitada. Essa estratégia de organização do processo ensinoaprendizagem articulado à realidade concreta e 
não apenas a um cenário montado, em que as situações são controladas pelo docente, propicia ao discente/profissional de saúde, além de capacitação meramente técnica, uma oportunidade que este se torne agente ativo das mudanças ${ }^{10}$.

Conforme a mesma autora ${ }^{10}$, a potencialidade desse processo de aprendizado, articulado à realidade do sistema de saúde, é levar o estudante/profissional de saúde a construir (e não apenas receber) o conhecimento a partir da articulação, "in loco", entre teoria e prática, a partir da experiência da interdisciplinaridade.

\section{Perguntas Iniciais}

A Epidemiologia, clássica em seus estudos de tecnologia leve-dura, demasiadamente voltados para os fluxos organizacionais da informação, muitas vezes dificulta sua incorporação pelos profissionais do serviço, distanciando-os de seus propósitos identificados com o comprometimento sanitário, causando assim uma relação fragilizada desta ciência com a assistência à saúde.

Assim, por vezes, a formulação de projetos de ação baseados nas reais necessidades do contexto em que o serviço se insere torna-se complexa, o que acaba por dificultar e desestimular a implantação de medidas efetivas, as quais iriam, potencialmente, solucionar problemas identificados. Esta dificuldade leva à formulação, muitas vezes, de programas verticais em resposta às necessidades singulares de cada realidade.

Nesse sentido, a experiência procura trazer a reflexão quanto à apreensão da importância da Epidemiologia enquanto ferramenta de processo de trabalho nos serviços de assistência à saúde, de modo a utilizá-la a favor do cuidado e organização de trabalho. Toda a condução do componente curricular, inquietações, visitas e projetos de intervenções foram pautados no questionamento: "Como os serviços de saúde têm se apropriado da Epidemiologia nas suas atividades e organização?”.

\section{Recuperação do processo vivido}

Foram visitadas as Unidades de Saúde de Unidades de Saúde de Parque das Dunas, Lagoa Seca e Felipe Camarão, todas do município de Natal. As Unidades de Parque das Dunas e Lagoa Seca apresentam em sua área de abrangência territórios com grande vulnerabilidade social e desenvolvem suas atividades na Estratégia Saúde da Família. Já a Unidade de Saúde de Felipe Camarão localiza-se em uma área mais favorecida com a presença de outros equipamentos sociais e de saúde, a qual conta com uma equipe multiprofissional e não pertence à estratégia saúde da família.

Ainda no momento da visita, cada grupo conversou com as equipes de saúde sobre um problema relacionado à Epidemiologia que eles julgavam relevante de ser aprofundado em termos de estratégia de enfrentamento. As problemáticas levantadas foram: necessidade de utilizar um mapa de situação de saúde (Unidade de Saúde Parque das Dunas), a necessidade de monitorar os excessivos acidentes de trânsito do bairro (Unidade de Saúde Lagoa Seca) e a necessidade de elaborar um fluxo para acompanhamento dos casos de hipertensão (Unidade de Saúde Felipe Camarão).

Posteriormente, o perfil epidemiológico construído pelos alunos foi apresentado pelos grupos em sala de aula. A partir das informações adquiridas e, com um problema eleito, cada grupo elaborou um projeto de intervenção para debater em sala de aula com a presença dos trabalhadores das equipes envolvidas.

Um grupo da Unidade de Saúde Parque das Dunas propôs a criação de uma sala de situação na qual os próprios usuários informassem uma 
ocorrência de casos de dengue, diagnóstico de hipertensão, entre outros. A ideia era de estimular a participação e notificar agravos de maior prevalência, levantados inicialmente em oficina com trabalhadores e usuários.

O grupo da Unidade de Saúde Lagoa Seca, ao perceber o grande número de hipertensos e um sistema de registro falho, propôs um instrumento de registro de casos e acompanhamento de tratamento, de modo a reorganizar o fluxo de informações e do próprio cuidado aos usuários que buscavam o serviço.

Ao final, o grupo da Unidade de Saúde Felipe Camarão traçou um plano de monitoramento dos acidentes de trânsito na área de abrangência da própria Unidade de Saúde. Partiu-se da ideia-força da vigilância, notificação e referência/contra referência.

\section{A reflexão de fundo}

Ao definir o perfil do profissional da área da saúde, as Diretrizes Curriculares Nacionais (DCN) parecem contemplar uma nova prática profissional, que pode ser realizada visando ultrapassar os limites dos consultórios particulares. Esse perfil aponta para uma formação generalista, humanista, crítica e reflexiva, capaz de atuar em todos os níveis de atenção, com base no rigor técnico e científico, dirigindo sua atuação para a transformação da realidade em benefício da sociedade ${ }^{11}$.

Essa experiência de partir da realidade do serviço para construção e aprofundamento do conhecimento e então propor intervenção sobre essa mesma realidade complexa, dinâmica e desafiadora, trouxe aos alunos do componente curricular de Epidemiologia em Saúde Coletiva uma maior aproximação do cotidiano do sistema de saúde brasileiro e com os desafios vividos diariamente pelos profissionais de saúde e das necessidades da população. Isso trouxe a perspectiva do descompasso entre a formação e a real necessidade do Sistema Único de Saúde (SUS).

Importante se faz que, a cada momento, a própria realidade deve ser o objeto do aprendizado, havendo lugar para o previsto e o imprevisto, o conhecido e o desconhecido, o já experimentado e o novo ${ }^{12}$. A vivência de participar do dia a dia de uma unidade de saúde proporcionou aos alunos a possibilidade de analisar criticamente não só o contexto no qual está inserida a unidade, mas também o processo de trabalho da equipe e sua própria formação.

Essa diversificação de cenário como uma estratégia para induzir mudanças mais profundas no processo de formação profissional é um elemento, em si mesmo, constitutivo de uma nova maneira de pensar esta formação ${ }^{10}$.

Para além da diversificação de cenário utilizando os serviços de saúde, foi importante e pertinente a proposta de tentar integrá-lo ao ensino, possibilitando assim, um retorno às contribuições feitas por quem está na ponta do sistema. Tal atitude pode, mais ainda, fortalecer o vínculo ensino-serviço, no intuito de uma construção melhor do conceito e de práticas em saúde, fazendo com que todos caminhem em uma mesma direção.

Quando há a integração ensino-serviço o discente se torna agente ativo no processo ensino-aprendizagem, tendo em vista a valorização dos seus conhecimentos, expectativas e experiências ${ }^{13}$. A formação engloba aspectos de produção de subjetividade, produção de habilidades técnicas e de pensamento e o adequado conhecimento do SUS. Ela deveria ter como objetivos a transformação das práticas profissionais e da própria organização do trabalho, e estruturar-se a partir da problematização do processo de trabalho e sua capacidade de dar acolhimento e cuidado às várias dimensões e necessidades de saúde das pessoas, dos coletivos e das populações ${ }^{14}$. 
Dessa maneira percebe-se um avanço no sentido de se quebrar a dicotomia saber-fazer, questionando-se assim o paradigma vigente. Com essa ruptura todos os atores se tornam sujeitos ativos em prol de condições de saúde melhores, baseada numa discussão que se inicia na academia e continua na prática, ou vice-versa, tendo nesse cenário uma pós-graduação ativa, presente e ciente das reais necessidades dos serviços, sensível aos seus verdadeiros nós críticos, podendo assim fazer discussões teóricas coerentes com o campo em que o programa se propõe a estudar.

Parte-se da premissa que a educação se dá para além do ambiente escolar, que ela se dá mesmo ao redor da escola ou da universidade, ela se apresenta "no sistema e na oposição a ele; na sala de aula em ordem, e no dia de greve estudantil; no trabalho rigoroso e persistente do professor-pesquisador e, ao mesmo tempo, no trabalho político do professor-militante" $"$.

Essas abordagens pedagógicas progressivas de ensino-aprendizagem, como a articulação ensino-serviço-comunidade, implicam uma prática pedagógica ética, crítica, reflexiva e transformadora, ultrapassando os limites do treinamento puramente técnico, para efetivamente alcançar a formação do homem como um ser histórico, inscrito na dialética da ação-reflexão-ação, tendo como consequência a formação de profissionais como sujeitos sociais ativos, e como tais detentores de competências éticas, políticas e técnicas e dotados de conhecimento, raciocínio, crítica, responsabilidade e sensibilidade para intervir nas questões sociais ${ }^{16}$.

\section{Os pontos de chegada}

Diante da pergunta inicial e da experiência vivida, acredita-se que a Epidemiologia tem alguns desafios a serem enfrentados quando se fala em assistência à saúde. Aparentemente, os profissionais dos serviços, em sua prática, utilizam de métodos e técnicas desta ciência à maneira como seu processo de trabalho permite. Não se pretende fazer juízo de valor, mas contribuir para uma otimização do que já é feito.

Com base no estudo realizado percebe-se que há uma apropriação, por parte dos profissionais, do perfil epidemiológico do território, uma clareza dos problemas existentes em nível de assistência e até mesmo de gestão. No entanto, existe uma lacuna no que diz respeito ao entendimento de conceitos, fluxos e ferramentas, que se impõem mais ao processo de trabalho.

Fica um tanto evidente a necessidade de ampliar a discussão no sentido de avaliar a situação e dar suporte para superar os obstáculos.

Ao avaliar o componente curricular, docente e discentes refletiram sobre como fortalecer essa aproximação da pós-graduação com os serviços de saúde. A articulação com o serviço, colocando seus profissionais como protagonistas nessa construção coletiva, foi fundamental para a sinalização das propostas de intervenção.

\section{CONSIDERAÇÕES FINAIS}

A proposta de organizar um componente curricular em prol das necessidades do serviço e dessa forma do estímulo a um raciocínio crítico do discente baseado em uma vivência prática de determinada realidade é algo um tanto desafiador para o contexto dos cursos Stricto Sensu. Extrapolar os muros da universidade e verificar, in loco, a realidade como ela é, ao mesmo tempo em que fomenta a construção do conhecimento, também retroalimenta o que se discute e teoriza em ambiente acadêmico, tendo em vista que a "vida real" pode causar situações inesperadas, as quais terão que ser trabalhadas, muitas vezes com um olhar diferente.

Diante das dificuldades percebidas no 
manejo da Epidemiologia como ferramenta que contribua para o processo de trabalho nos serviços, percebe-se a importância da universidade, enquanto instituição formadora, como capaz de auxiliar na utilização dessa ciência.

A aproximação da academia com os serviços, a partir dos programas de pósgraduação stricto sensu, pode contribuir no sentido de integrar, bem como fortalecer a teoria discutida com a prática vivida, aliando assim, discussões com a realidade local ${ }^{17}$.

Inquietações, com certeza, surgirão da realidade dos serviços e poderão ser discutidas academicamente de maneira mais efetiva. Isso fortalece o vínculo e comunicação entre os diversos profissionais (docentes, discentes, trabalhadores de saúde do serviço), refletindo em ações e formulação de projetos coerentes com as necessidades de cada território.

Aponta-se também uma limitação da experiência, a qual se configura na elaboração de propostas de projetos por parte dos discentes, os quais, provavelmente não serão aplicados à realidade. Tal fato deve-se, principalmente, ao pouco tempo do componente curricular.

Contudo, essa limitação gerou discussões na tentativa de solucioná-la. A proposta é que futuras ofertas do componente curricular pelo Programa de Pós-graduação em Saúde Coletiva prevejam vagas para profissionais do serviço que desejem cursá-lo como aluno especial. Essas vagas serão pactuadas com a Secretaria Municipal de Saúde de Natal promovendo um fortalecimento da aproximação pós-graduação e serviços de saúde, permitindo que se tracem planos cada vez mais condizentes com a realidade vivenciada nos territórios e contribuições efetivas para transformação da prática dos serviços.

A experiência mostrou-se muito proveitosa, uma vez que foram identificadas claramente as dificuldades referentes à utilização da Epidemiologia nos serviços. Assim, tornaram-se visíveis para que a academia se sensibilize e consiga trabalhar aspectos teóricos de maneira mais palpável à utilização dessa ciência, por vezes considerada como dura, por parte dos profissionais que estão na ponta do sistema.

É importante ressaltar que não há pretensão de que um único componente curricular seja capaz de detectar problemas a serem resolvidos com soluções prontas. O destaque é para a sensibilização que a academia deve ter em seu compromisso com a sociedade e o SUS.

O desafio da integração entre universidade e serviço de saúde é elemento fundamental no avanço de políticas públicas de saúde, em especial na perspectiva dos cursos de pósgraduação stricto sensu, responsáveis pela produção de novo conhecimento e pelo entendimento do serviço como cenário privilegiado para aprendizado do SUS. Pretendese valorizar o serviço em todas as suas potencialidades e a perspectiva que a academia se integre nesta construção, para juntos, unindo saberes, poder-se "ser mais" e oferecer um cuidado equânime, digno, o qual a população merece e tem direito.

\section{ABSTRACT \\ Epidemiology in Collective Health: reflections on a pedagogical experience integrating postgraduation and health service}

The epidemiology supports the health planning showing itself as an essential knowledge in decision making and knowledge production. This study aims to reflect about a pedagogical experience started by the curricular content named Epidemiology in Public Health at the Public Health Postgraduate Program in Universidade Federal do Rio Grande do Norte. It is an experience report which was developed based on Holliday's theoretical principles of systematization of experiences. This experience was focused on a pedagogical strategy that saw 
the service situation as a place for constructing a proposed intervention, allowing the student the protagonism as a fundamental element of his learning. The articulation with the service and the staff was essential for signaling the proposed intervention. The challenge of integration between universities and the health service is a fundamental element in the advancement of public health policies, particularly in the view of responsible postgraduate courses for the production of new knowledge and for the understanding of the service as a privileged scenario for the learning of the Unified Health System.

Descriptors: Epidemiology. Postgraduate Education. Unified Health System. Teaching Care Integration Services.

\section{REFERÊNCIAS}

1. Nunes ED. A saúde coletiva: história de uma ideia e de um conceito. Saúde Soc. 1994; 3(2):5-21.

2. Bosi, MLM, Prado SD. Alimentação e Nutrição em Saúde Coletiva: constituição, contornos e estatuto científico. Ciênc Saúde Coletiva. 2011; 16(1):7-17.

3. Minayo MCS. Atuação da ABRASCO em relação ao ensino de Pós-graduação na área de Saúde Coletiva. In: Lima NT, Santana JP (Org). Saúde Coletiva como compromisso: A história da ABRASCO. Rio de Janeiro: FIOCRUZ, 2006. 232 p.

4. Nunes ED; Ferreto LE, Barros NF. A pósgraduação em Saúde Coletiva no Brasil: trajetória. Ciênc Saúde Coletiva. 2010; 15(4):1923-34.

5. Silva EM, Costa ICC, Lima KC. Da Odontologia Social à Saúde Coletiva: uma história de 37 anos na Universidade Federal do Rio Grande do Norte. Rev Ciênc Plur. 2015; 1(2):1-3.

6. Cyrino EG, Toralles-Pereira ML. Trabalhando com estratégias de ensinoaprendizado por descoberta na área da saúde: a problematização e a aprendizagem baseada em problemas. Cad Saúde Pública. 2004; 20(3):780-8.

7. Turci SRB, Guilam MCR, Câmara MCC. Epidemiologia e Saúde Coletiva: tendências da produção epidemiológica brasileira quanto ao volume, indexação e áreas de investigação - 2001 a 2006. Ciênc Saúde Coletiva. 2010; 15(4):1967-76.

8. Holliday OJ. Para sistematizar experiências. $2^{a}$ Ed. Brasília: MMA, 2006. 128 p.

9. Freire P. Pedagogia da autonomia: saberes necessários à prática educativa. $36^{\mathrm{a}}$ Ed. São Paulo: Paz e Terra, 2009. 148 p.

10. Feuerwerker, LCM. A construção de sujeitos no processo de mudança da formação dos profissionais da saúde. Divulg Saúde Deb. 2000; 22:18-24.

11. Haddad AE, Morita MC, Pierantoni CR, Brenelli SL, Passarella T, Campos FE. Formação de profissionais de saúde no Brasil: uma análise no período de 1991 a 2008. Rev Saúde Pública. 2010; 44(3):38393.

12. Werneck MAF, Senna MIB, Drumond MM, Lucas SD. Nem tudo é estágio: contribuições para o debate. Ciênc Saúde Coletiva. 2010; 15(1):221-31.

13. Souza AL, Carcereri DL. Estudo qualitativo da integração ensino-serviço em um curso de graduação em Odontologia. Interface Comunic Saúde Educ. 2011; 15(39):107184.

14. Ceccim, RB, Feuerwerker LCM. O quadrilátero da formação para a área da saúde: ensino, gestão, atenção e controle social. Rev Saúde Coletiva. 2004; 14(1):4165.

15. Brandão CR. O que é educação. $49^{a}$ Ed. São Paulo: Brasiliense, 2007. 116 p.

16. Mitre SM, Siqueira-Batista R, Girardi-deMendonça JM, Morais-Pinto NM, Meirelles 
CAB, Pinto-Porto C, Moreira T, Hoffmann LMA. Metodologias ativas de ensinoaprendizagem na formação profissional em saúde: debates atuais. Ciênc Saúde Coletiva. 2008; 13(sup. 2):2133-44.

17. Neta AA, Feitosa MSC. A comunidade como local de protagonismo na integração ensino-serviço e atuação multiprofissional. Trab Educ Saúde. 2016; 14(1): 22135 .
Correspondência para:

Luiz Roberto Augusto Noro

e-mail: noro@ufrnet.br

Universidade Federal do Rio Grande do Norte

Departamento de Odontologia / Programa de Pós-graduação em Saúde Coletiva

Avenida Salgado Filho, 1787

59056-000 Natal/RN 